\title{
Evaluation of Independent Component Analysis Algorithms for Electroencephalography Source Separation
}

\author{
Masoud Zakeri and Zohreh Zakeri \\ School of Electronic, Electrical \& Computer Engineering, University of Birmingham, UK \\ $\{m x z 848$, zxz849\}@bham.ac.uk
}

\begin{abstract}
Since each ICA algorithm employs a different approach for source estimation, the result of the estimated sources could be changed. The proposed evaluation method applies three different ICA algorithms on EEG datasets including FastICA, Infomax and Extended-Infomax algorithms. The analysis demonstrates that different ICA algorithms do not have a significant effect on the accuracy of the Support Vector Machine (SVM) classifier in detecting right and left hand imagery movements.
\end{abstract}

Keywords: Independent Component Analysis, EEG, Artefact rejection.

\section{Introduction}

Electroencephalography (EEG) is a measure of the brain's electrical activity that can be recorded by placing electrodes on the scalp [1]. It can explore brain function, which is useful for cognitive processing in clinical applications. The EEG brain signals are linearly mixed with non-brain signals due to volume conduction [2]. In order to study brain function, it is desired to remove non-brain signals from the EEG data such as eye movement artefacts.

Among EEG artefact rejection methods, Independent Component Analysis (ICA) is the most commonly used method for EEG source separation and artefact rejection. ICA is a statistical method that is able to separate the brain and non-brain signals from the observed EEG data [3]. It belongs to Blind Source Separation (BSS) methods and can estimate underlying sources of EEG data, which are temporally independent. Several ICA algorithms exist to decompose EEG data into independent components such as FastICA, Infomax and Extended-Infomax. The approach of each ICA algorithm is different in estimating independent components; hence the results of decompositions and artifacts by each ICA algorithm may be different. FastICA is based on a fixed-point iteration scheme that attempts to maximize non-Gaussian properties of the recovered components' distributions. Negentropy is employed to measure the non-Gaussianity and independence of components. Infomax and Extended-Infomax are natural gradient-based algorithms that minimise the mutual information of the component probability distributions. The difference between Info-max and Extended-Infomax is the type of the component distribution that can be estimated by each of them. Infomax is able to separate source signal with 
super-Gaussian distribution (positive kurtosis), while Extended-Infomax can separate sources with both sub-Gaussian (negative kurtosis) and super-Gaussian distributions.

In the scope of this research, the performance of three common ICA algorithms, FastICA [4], Infomax [5], and Extended-Infomax [6], in terms of increasing the accuracy of the Support Vector machine (SVM) classifier [7] for motor imagery task detection will be analysed.

\section{Method}

\subsection{EEG Data}

The raw EEG dataset has been provided by 2008 BCI competition IV, Data set II [8]. The data was recorded from 9 subjects while executing four motor imagery tasks: left and right hand imagined movements. The EEG signals were sampled at $250 \mathrm{~Hz}$ and filtered by a $0.5-100 \mathrm{~Hz}$ band-pass filter with and a $50 \mathrm{~Hz}$ notch filter. $\mathrm{C} 3, \mathrm{Cz}$ and $\mathrm{C} 4$ are three EEG electrodes that are used to record the data according to the 10-20 international electrodes position [9]. At the beginning of the trials, subjects were shown a fixation cross on the computer screen. Subsequently, different of eye movements of blinking, rolling, up-down or left-right movements were instructed to the subjects. Subjects have to imagine the corresponding hand movements over a period of 4 seconds. A short break was considered between each trial.

\subsection{Data Analysis}

Three selected ICA algorithms including FastICA, Infomax and Extended-Infomax have been applied to the data to separate EEG brain and non-brain sources from the EEG mixture. The patterns of EEG signal artefacts associated to the different eye movements including blinks, vertical and horizontal eye movements have been obtained by ICA decompositions and removed from the recordings.

The EEG data channels have been split into epochs of 1 second with $90 \%$ overlap. Fast Fourier Transform (FFT) is used to extract features of delta, theta, alpha and beta frequency bands of 1-4 Hz, 4-8 Hz, 8-13 Hz and 13-20 Hz, respectively. Then, other features including Root Mean Square (RMS) amplitude, kurtosis, skewness, average power, minimum amplitude, variance and peak to peak values of the signal are extracted. Also, Principal Component Analysis (PCA) [10] has been used to reduce the dimension of the feature vector for avoiding the effect of noise and outliers in to the classifier.

\subsection{Classifier}

SVM classifier is applied to distinguish the left and right hand imagery movements. The SVM classifier is trained on a sub-space of the data using a set of extracted features. In order to evaluate the performance of the trained SVM classifier, a 10-fold cross validation method is employed. 


\section{Results and Discussion}

The effect of the FastICA, Infomax and Extended-Infomax algorithms on the accuracy of SVM classifier for motor imagery task detection is shown in Figure 1. On one hand, the accuracy of the SVM classifier is different for each individual EEG data. The between subject difference can be due to the distinctness of subjects from whom EEG data was collected and different conditions of each individual. On the other hand, the variability of the SVM classifier's accuracy is small across ICA algorithms for each individual EEG data.

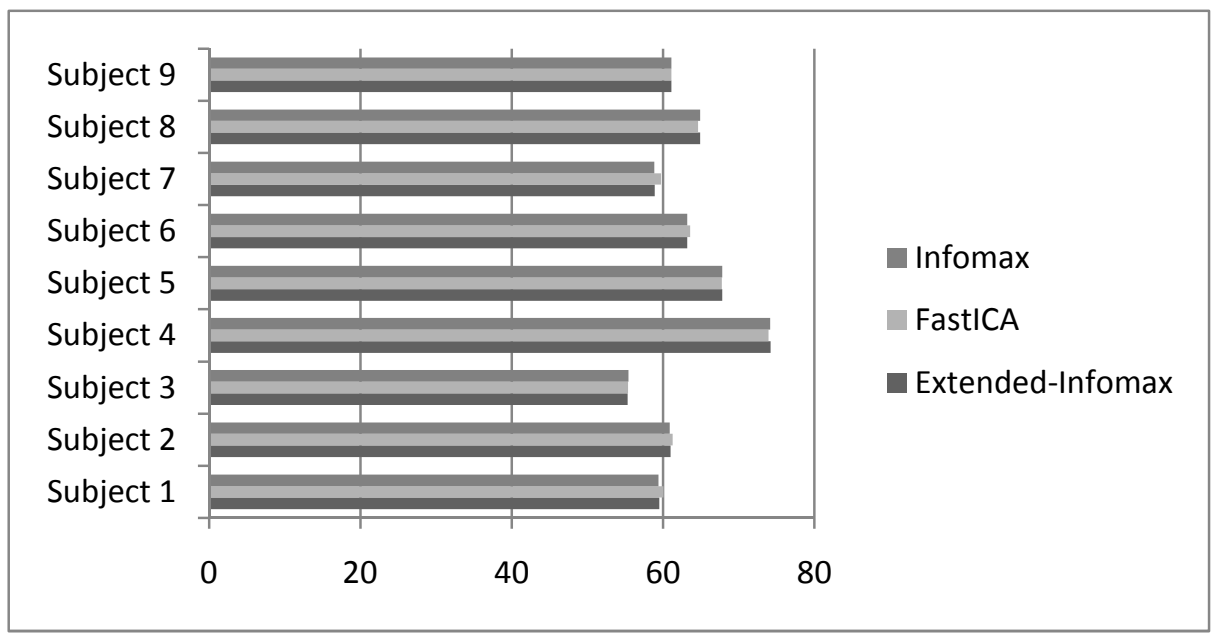

Fig. 1. The performance of SVM classifier method using three different ICA algorithms of FastICA, Infomax and Extended-Infoamx

The Analysis of Variance (ANOVA) test at 5\% significance level represents no significant different between ICA algorithms' effect on the accuracy of SVM classifier performance. Although the selected ICA algorithms employ different approaches of source estimation, they are able to estimate and separate eye related components from EEG data. The eye movement components can be detected and separated as single individual sources and removed from the EEG mixture. The difference between ICA algorithms implementations does not affect the performance of SVM classifier to classify right and left imagery movements.

\section{Conclusion}

In this study, the accuracy of the SVM classifier to distinguish the left and right hand imagery movements are assessed. The imagery movements are distinguished by SVM with no significant difference in the accuracy of the classifier regardless of using different ICA algorithms in the EEG data pre-processing. Different ICA algorithms 
are applied to pre-process the EEG data and eye movement artifact rejection has no significant effect on the performance on SVM classifier. They all can detect and separate bran non-brain sources from EEG mixture and clean the data to be given to the classifier method. Further studies can be done on the comparison of the effect of ICA algorithms and other artifact rejection methods on classification method's accuracy.

\section{References}

1. Berger, H.: Über das elektrenkephalogramm des menschen. European Archives of Psychiatry and Clinical Neuroscience 87(1), 527-570 (1929)

2. Sanei, S., Chambers, J.A.: EEG signal processing. Wiley-Interscience (2008)

3. Comon, P.: Independent component analysis, a new concept? Signal Processing 36(3), 287-314 (1994)

4. Hyvarinen, A.: Fast and robust fixed-point algorithms for independent component analysis. IEEE Transactions on Neural Networks 10(3), 626-634 (1999)

5. Cardoso, J.-F.: Infomax and maximum likelihood for blind source separation. IEEE Signal Processing Letters 4(4), 112-114 (1997)

6. Lee, T.-W., et al.: A unifying information-theoretic framework for independent component analysis. Computers \& Mathematics with Applications 39(11), 1-21 (2000)

7. Suykens, J.A., Vandewalle, J.: Least squares support vector machine classifiers. Neural Processing Letters 9(3), 293-300 (1999)

8. Leeb, R., et al.: BCI Competition 2008-Graz data set B. Graz University of Technology, Austria (2008)

9. Homan, R.W., Herman, J., Purdy, P.: Cerebral location of international 10-20 system electrode placement. Electroencephalography and Clinical Neurophysiology 66(4), 376-382 (1987)

10. Jolliffe, I.T.: Principal component analysis, vol. 487. Springer, New York (1986) 